\title{
Empirical pseudo-potential studies on electronic structure of semiconducting quantum dots
}

\author{
ANJALI KSHIRSAGAR* and NEELESH KUMBHOJKAR \\ Department of Physics, University of Pune, Pune 411 007, India
}

\begin{abstract}
Theoretical investigations of electronic structure of quantum dots is of current interest in nanophase materials. Empirical theories such as effective mass approximation, tight binding methods and empirical pseudo-potential method are capable of explaining the experimentally observed optical properties. We employ the empirical pseudo-potential to calculate the gap between the highest occupied molecular orbital (HOMO) and the lowest unoccupied molecular orbital (LUMO) as a function of shape and size of the quantum dots. Our studies explain the building up of the bulk band structure when the size of the dot is much larger than the bulk Bohr exciton radius. We present our investigations of HOMO-LUMO gap variation with size, for CdSe, ZnSe and GaAs quantum dots. The calculated excitonic energies are sensitive to the shape and size of quantum dots and are in good agreement with experimental HOMO-LUMO gaps for CdSe quantum dots. The agreement improves as experimentally observed lattice contraction is incorporated in pseudo-potential calculations for $\mathrm{ZnSe}$ quantum dots. Electronic structure evolution, as the size of quantum dot increases, is presented for CdSe, ZnSe and GaAs quantum dots.
\end{abstract}

Keywords. Quantum dots; empirical pseudo-potential; electronic structure; HOMO-LUMO gap.

\section{Introduction}

Quantum dots serve as very attractive and interesting objects for scientific research of three-dimensionally confined systems. The experimental and theoretical progress has opened up a new realm of fundamental physics and chemistry as researchers can make and study artificial analogues of atoms, molecules and crystals. Research of more than two decades has given a way to tailor materials having desired optical and electronic properties (Yoffe 1993). The main focus of the research in this field, is to investigate, experimentally as well as theoretically, the effect of three-dimensional confinement on the electronic structure which is affected by quantum size effects, dielectric confinement effects and high surface to volume ratio for semi-conducting quantum dot. Variation in the optical and electronic properties is substantial for semiconductors as compared to that for the metals. The clusters of metals start showing bulk-like properties for comparatively small sizes (with few hundred atoms) as against semiconductors (where even with few thousand atoms, the bulk like states do not show up). This can be explained by considering the bands of solids which are centred about the atomic energies with the width of the band related to the strength of the nearest neighbour interactions. As the size of the clusters increases, centre of the band develops first and the edges develop last. In metals, the Fermi energy lies amidst the bands and the relevant

*Author for correspondence (anjali@physics.unipune.ernet.in) energy spacing is small at temperatures above a few Kelvin. As opposed to this, in semiconductors, Fermi energy lies between two bands, hence the edges of the band determine the low-energy optical properties and across the gap, the states are strongly size-dependent even when the crystallites contain few thousand atoms.

Various $a b$ initio and empirical theoretical methods are being used for understanding the size dependent optical properties of semi-conducting quantum dots. Ab initio as well as empirical tight binding methods (TB), effective mass approximation (EMA), empirical pseudo-potential method (EPM) and effective bond orbital model (EBOM) are some of the important methods that are extensively applied in this field to understand the electronic structure and related properties, the optical properties, especially the bandgap variation with size, etc. Lee et al (2001) calculated the eigenvalues and electron addition energy or chemical potential variation, using Kohm-Sham-local density approximation (KS-LDA) for an $N$-electron system $(N=1, \ldots, 12)$ as a function of the ellipticity of the dot. Troparevsky and Chelikowsky (2001) employed finite difference pseudo-potential method in real space to calculate the gaps between the highest occupied molecular orbital (HOMO) and the lowest unoccupied molecular orbital (LUMO), binding energies, and polarizabilities of $\mathrm{Cd}_{n} \mathrm{X}_{n}(\mathrm{X}=\mathrm{S}$, Se and $n=2$ to 8). Matxain et al (2001) estimated the excitation energies for $\mathrm{Zn}_{i} \mathrm{~S}_{i}(i=1$ to 9 ) using time-dependent density functional theory (TD-DFT) and concluded that excitation occurs from non-bonding $p$ orbitals of sulphur. Vasiliev et al (1999) employed TDDFT formalism within local density approximation 
(LDA) to calculate absorption spectra of $\mathrm{Ga}_{n} \mathrm{As}_{m}$ clusters $(n+m \leq 10)$ and have attributed the long absorption tails, extending deep into lower transition energies, to free surfaces in clusters. Joswig et al (2000) studied structural and electronic properties and stability of stoichiometric $\mathrm{Cd}_{n} \mathrm{~S}_{n}$ for $n$ ranging up to 100 for both $\mathrm{Cd}$ and $\mathrm{S}$, using a simplified linear combination of atomic orbitals (LCAO) DFT-LDA scheme. Eichkorn and Ahlrichs (1998) studied smaller CdSe clusters with surfactants, by DFT formalism. It may be noticed that the first principles methods have been restricted to work for clusters containing few tens of atoms only. On the other hand, experimental observations are available for nano-particles in the size range of a few nanometers. Thus, to compare theoretical results with experimental data, one has to take recourse of empirical methods.

In simple empirical methods, experimental data is used to adjust and fix electronic structure parameters whereas first principles methods need no experimental data but attempt to support the experimental observations by self consistent calculations. Present computational power does not allow use of $a b$ initio methods for experimentally synthesized size range of nano-crystallites, as they become computationally demanding. Accurate $a b$ initio calculations based on DFT suffer an additional drawback of underestimating the optical gaps due to the use of LDA. It is well known that bandgap in semiconductors are underestimated by up to $30-50 \%$ within LDA. One has, therefore, to resort to empirical models that can explain not only qualitatively but also quantitatively, the size dependence of electronic structure of these materials. Empirical theories, based on certain parameters derivable either from experiments or from accurate theoretical calculations are more popular in predicting various properties of quantum-confined structures. Calculations based on tight binding theories have been found to be reliable for semiconducting quantum dots up to few hundred atoms (Lippens and Lannoo 1989; Wang and Herron 1990a, b; Nair et al 1992; Ramaniah and Nair 1993, 1995). Ramaniah and Nair (1993, 1995) employed tight binding method to calculate the inter-band transitions of cubic $\mathrm{CdS}$ and CdSe quantum dots in the size range 10-60 $\AA$ and GaAs quantum dots in the size regime $8-28 \AA$. They have established correspondence between TB and multiband EMA energy levels to test the applicability of the selection rules of the latter technique. The zinc blende structure of these semiconductors is replaced by inversion symmetric $f c c$ lattice, hence the model does not distinguish between these two structures. Leung et al (1998) studied the excitonic fine structure in CdSe nanocrystals incorporating electron-hole and spin-orbit interactions within tight binding model. They predict existence of a 'dark' predominantly triplet band-edge excitonic state with finite life time. They also warrant a more realistic model for surface passivation and band mixing. Pokrant and Whaley (1999) studied CdSe nanoparticles using TB model incorporating the effect of organic capping ligands and inorganic capping shells, surface reconstruction and shape information from experiments. Their studies emphasize the dependence of electronic and optical properties on the shape of nanocrystals. They have shown that when the surface is saturated with shells of inorganic species like $\mathrm{CdS}$, a differential localization of the valence and conduction band edge states results. Over last two decades or so, TB methods have received considerable attention, both because of their intuitive simplicity and their realistic description of structural and dielectric properties in terms of chemical bonds. Since the method uses a smaller basis set, one can study larger nanoparticles. The Slater-Koster (Slater and Koster 1954) description to treat TB approach as an interpolation scheme has been extensively used for metals and semiconductors. Sapra and co-workers (Sapra and Sarma 2004; Viswanatha et al 2005) extensively studied the size dependence of HOMO-LUMO gap and variations in the valence band maximum and conduction band minimum for almost all III-V and II-VI semiconductor compounds. They demonstrated the importance of inclusion of anion and cation $d^{5}$ states in the basis and inclusion of next near neighbour interactions in the TB Hamiltonian to correctly reproduce the first principles electronic structure of these materials.

As a consequence of the computational ease, conceptual simplicity and their predictive powers, several other approaches and improvements in these empirical theories are also proposed (Nair et al 1987; Kayanuma 1988; Ruppin 1989; Kayanuma and Momiji 1990; Sercel and Vahala 1990; Tran Thoi et al 1990). These methods are based on an intelligent guess for the wave-functions to achieve the desired accuracy. The trial wave-functions could either be written as the product of solutions for non-interacting particles involving lowest order Bessel function as done in EMA or an exponent function taking its form from $1 \mathrm{~s}$ hydrogen-like wave-function.

We have studied the shape and size dependence of the electronic and optical properties of II-VI semiconducting quantum dots having sizes from $7 \AA$ onwards (Kumbhojkar 2002). A detailed analysis of the quantum size effects (QSE) in II-VI zinc blende quantum dots, viz. (Cd and Zn)S (Kumbhojkar and Kshirsagar 2001a) and (Cd and Zn)Se (Kumbhojkar and Kshirsagar 2001b) has been carried out. We have successfully shown that empirical pseudopotential method can estimate the sizes of quantum dots given the excitation energies (Kumbhojkar et al 2000).

In the present paper, we present our results of electronic structure calculations for $\mathrm{CdSe}, \mathrm{ZnSe}$ and $\mathrm{GaAs}$, based on empirical pseudo-potential method (EPM), modified for quantum dots. II-VI Semiconductors and their quantum dots commonly exist in zinc blende or wurtzite structure. We have experimentally synthesized $\mathrm{ZnS}$ and $\mathrm{ZnSe}$ quantum dots using chemical routes and they revealed zinc blende structures. Therefore, we present our theoretical results for this phase only. Experimental ob- 
servation of lattice contraction has also been accounted for in EPM calculations. The method is also capable of predicting the trends in electronic structure evolution from cluster to bulk. GaAs has also been investigated on similar lines as a prototype III-V semiconductor to establish the applicability of the present method to this class of semiconductor quantum dots also.

\section{Methodology and computational details}

The electrons in a solid can be separated into two categories: itinerant or valence electrons and core electrons. The itinerant electrons in the solids are responsible for most of their chemical properties. The core electrons are strongly localized near the nucleus and have negligible overlap with the electrons from the neighbouring atoms. They are thus chemically inert and do not affect the chemical bonding. They can, therefore, be excluded from the electronic structure calculations performed to study the bonding properties and properties governed by the states near the valence band maximum and the conduction band minimum. This fact can be extended for calculations of electronic properties of quantum dots. In the 'frozen core approximation', the core states of the bulk or quantum dot are taken to be identical to those of the isolated atoms.

Pseudo-potentials eliminate the atomic core states and the strong potentials that are used to bind them (Harrison 1966). Hence, the computational efforts to solve the Hamiltonian corresponding to many electron system, are significantly reduced for the valence electrons. The effect of the core electrons and nuclei is replaced by an effective 'pseudo-potential', as shown in figure 1. This potential is chosen as soft as possible and the nodeless pseudo wavefunctions are matched with the actual electronic orbitals beyond a certain distance, $r_{\mathrm{c}}$, often referred to as the critical radius. Pseudo-potentials can be constructed either by ab initio approach (Pickett 1989) or by empirical approach (Cohen and Heine 1970; Cohen and Chelikowsky 1989). We have employed the elegant yet simple empirical approach and we briefly discuss the modifications to employ them for quantum dots (Rama Krishna and Friesner 1991a, b, 1992).

In pseudo-potential method, we solve the following one-electron equation for a valence electron in the state with wave-vector, $\vec{k}$ and band index, $n$

$$
\left[-\frac{1}{2} \nabla^{2}+V_{\mathrm{ps}}(\vec{r})\right] \psi_{\vec{k}}^{n}(\vec{r})=\varepsilon_{\vec{k}, n} \psi_{\vec{k}}^{n}(\vec{r}),
$$

where atomic units ( $\hbar=m=e^{2}=1$ numerically) are used throughout; energy is measured in Hartree in a.u. $V_{\mathrm{ps}}(\vec{r})$ is the crystal pseudo-potential and contains contributions from all the atoms in the crystal. Expanding the electronic wave-function, $\psi_{\vec{k}}^{n}$, in terms of plane waves

$$
\psi_{\vec{k}}^{n}(\vec{r})=\sum_{\vec{G}} c_{\vec{k}+\vec{G}}^{n} e^{i(\vec{k}+\vec{G}) \cdot \vec{r}},
$$

$\vec{G}$ being the reciprocal lattice vectors; we get to solve following matrix equation

$$
\left[H_{\vec{k}}^{\vec{G} \vec{G}^{\prime}}-\varepsilon_{\vec{k}, n}\right] c_{\vec{k}+\vec{G}}^{n}=0,
$$

where

$$
H_{\vec{k}}^{\vec{G} \vec{G}^{\prime}}=\Omega|\vec{k}+\vec{G}|^{2}+V_{\mathrm{ps}}^{\vec{G} \vec{G}^{\prime}} .
$$

Here, $V_{\mathrm{ps}}^{\vec{G} \vec{G}^{\prime}}$ are the Fourier transforms of the pseudopotential, $V_{\mathrm{ps}}(\vec{r})$. These can be obtained from atomic form factors and structure factors since the crystal pseudopotential, $V_{\mathrm{ps}}(\vec{r})$, is written in terms of the atomic pseudopotentials of the constituents, as follows

$$
V_{\mathrm{ps}}(\vec{r})=\sum_{\vec{R}, j} V_{p}^{j}\left(\vec{r}-\vec{R}-\delta_{j}\right),
$$

where $V_{p}^{j}$ is the atomic pseudo-potential of the $j$ th basis atom situated at $\delta_{j}$ from the corner of the unit cell, and $\vec{R}$ is the position vector of the corner of the unit cell from arbitrary origin. Since the atomic pseudo-potentials are

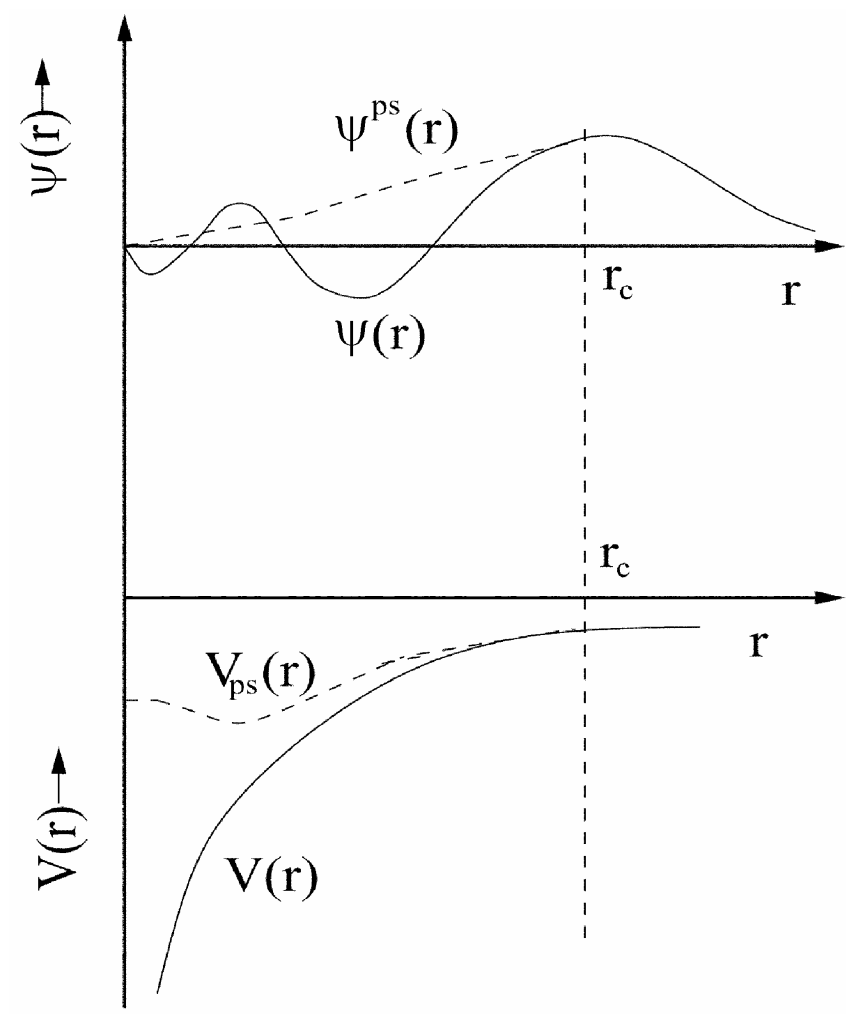

Figure 1. Schematic diagram showing the replacement of actual atomic potential, $V(\vec{r})$, by the pseudo-potential, $V_{\mathrm{ps}}(\vec{r})$ and the actual all electron wave-function, $\psi(\vec{r})$, by pseudo wave-function, $\psi^{\mathrm{ps}}(\vec{r})$. They match beyond a critical radius, $r_{\mathrm{c}}$. 
spherically symmetric, it turns out that the form factors, $v_{j}(\vec{G})$, of the atomic pseudo-potentials depend only on the magnitude of the reciprocal lattice vector, $\vec{G}$ and the number of form factors needed to represent the potential correctly is reduced considerably. The structure factor gives position of pseudo-potential within the crystal whereas the form factors are the measure of scattering. The structure factors can be calculated using X-ray diffraction analysis of the crystal, from the underlying geometry of the structure. The Fourier components of the potential are evaluated from the available experimental data. In case of semiconductors, photo-emission and reflectivity data can be used to calculate the pseudopotential form factors, and for metals the Fermi surface data can also be used. These form factors can be adjusted with the help of experimental data such as optical spectra, to reproduce the bulk band structure accurately. Pseudopotential is generally chosen to be so 'soft' that it does not contain any bound state. The pseudo-potential for the crystal is generated from atomic pseudo-potentials and the matrix (3) is solved to get the electronic structure of valence electrons. The atomic pseudo-potential can further be divided into local part, $V_{\mathrm{L}}$, and a non local, $l$ dependent part, $V_{\mathrm{NL}}$. Though the inclusion of non-local part makes the pseudo-potential much more accurate and applicable over a wide energy range, we have employed only the local part in the present study as it is sufficiently accurate to explain the gross features of the band structure. The local part of the empirical pseudo-potential may be written in terms of symmetric and anti-symmetric parts of the structure factors and those of pseudo-potential form factors

$$
V_{\mathrm{L}}(\vec{r})=\sum_{\vec{G}}\left[V_{\mathrm{S}}(\vec{G}) S_{\mathrm{S}}(\vec{G})+i S_{\mathrm{A}}(\vec{G}) V_{\mathrm{A}}(\vec{G})\right] e^{i \vec{G} \cdot \vec{r}} .
$$

It is well known that most of the semiconductors exist in zinc blende or wurtzite structure. The zinc blende structure is composed of two inter-penetrating $f c c$ structures with different basis atoms on each lattice. If we assume the origin in between these two atoms, then for a binary compound, the symmetric and anti-symmetric parts of the Fourier components of potential may be written as

$$
\begin{aligned}
& V_{\mathrm{S}}(\vec{G})=\frac{1}{2}\left[v_{1}(\vec{G})+v_{2}(\vec{G})\right], \\
& V_{\mathrm{A}}(\vec{G})=\frac{1}{2}\left[v_{1}(\vec{G})-v_{2}(\vec{G})\right],
\end{aligned}
$$

where $v_{1}(\vec{G})$ and $v_{2}(\vec{G})$ are the pseudo-potential form factors for individual atoms. The symmetric and antisymmetric parts of the structure factors for each atom may be written as

$$
\begin{aligned}
& S_{\mathrm{S}}(\vec{G})=\cos \left(\vec{G} \cdot \vec{t}_{1}\right), \\
& S_{\mathrm{A}}(\vec{G})=\sin \left(\vec{G} \cdot \vec{t}_{1}\right),
\end{aligned}
$$

where $\vec{t}_{1}$ and $\vec{t}_{2}$ are the positions of first and second atom, respectively. $\vec{t}_{1}=-\vec{t}_{2}$ if the origin is situated in between the two atoms. The reciprocal vectors are defined, in terms of the lattice constant, $a_{0}$, of the material as

$$
\vec{G}=\frac{2 \pi}{a_{0}}\left[G_{x}, G_{y}, G_{z}\right] .
$$

Substituting (11) into (9) and (10), we get

$$
\begin{aligned}
& S_{\mathrm{S}}(\vec{G})=\cos \left[\frac{\pi}{4}\left(G_{x}+G_{y}+G_{z}\right)\right], \\
& S_{\mathrm{A}}(\vec{G})=\sin \left[\frac{\pi}{4}\left(G_{x}+G_{y}+G_{z}\right)\right] .
\end{aligned}
$$

Thus knowing the Fourier transform of atomic pseudopotentials for a given $\vec{G}$ shell, it is quite straight forward to generate the pseudo-potential matrix elements in the basis of plane waves. The best choice for expanding electronic wave-functions using pseudo-potentials is plane waves. The main advantages of using plane waves as the basis are that, they are origin independent, are not attached to a particular atom and the expansion coefficients are not coupled. However, since plane waves treat all regions in space with equal precision irrespective of whether the wave function varies slowly or rapidly in a given region, this affects the precision of the calculation. Since electron is bound in a solid and plane waves represent a free particle, number of plane waves required to represent the electronic wave-function, perfectly converged, in a solid may be large depending on the electron energy. However, plane wave pseudo-potential method is still preferred for the determination of electronic structure for clusters due to its simplicity and the advantages listed above. In the next section, we describe the modifications of the electronic structure method for quantum dots as implemented in the present work.

\subsection{Modification for semiconducting quantum dots}

The reduced dimensionality of the semiconducting quantum dots often results into discretization of energy states giving rise to molecular like orbital energies instead of band structure. We can obtain these energy levels by imposing proper boundary conditions on the wave functions. In the present work, empirical pseudo-potentials which work for bulk materials are used for predicting size quantization effects in quantum dots. This is possible because experimental evidences such as transmission electron microscopy and X-ray diffraction studies have shown that nano-crystallites have same geometric structure as that of the bulk. The bulk pseudo-potentials are generated by using form factors reported by Cohen and Bergstresser (1966) that are accurate to 0.01 Ry. Table 1 lists the form factors used to generate the pseudo-potentials in the present study. These form factors can be adjusted with the 
Table 1. Pseudo-potential form factors, in Hartree a.u., used to calculate the electronic structure in the present study.

\begin{tabular}{lccccccc}
\hline Material & $V_{3}^{\mathrm{S}}$ & $V_{8}^{\mathrm{S}}$ & $V_{11}^{\mathrm{S}}$ & $V_{3}^{\mathrm{A}}$ & $V_{4}^{\mathrm{A}}$ & $V_{11}^{\mathrm{A}}$ & $V_{12}^{\mathrm{A}}$ \\
\hline ZnSe (Cohen et al 1966) & $-0 \cdot 115$ & $+0 \cdot 050$ & +0.030 & $+0 \cdot 090$ & $+0 \cdot 060$ & 0.015 & \\
CdSe (Rama Krishna et al 1991) & $-0 \cdot 150$ & +0.005 & +0.020 & $+0 \cdot 095$ & $+0 \cdot 060$ & 0.025 & 0.025 \\
GaAs (Cohen et al 1966) & -0.115 & +0.005 & +0.030 & +0.035 & +0.025 & 0.005 & \\
\hline
\end{tabular}

Hartree a.u. $=27 \cdot 2 \mathrm{eV}$

help of experimental data such as optical spectra, to reproduce the bulk band structure accurately.

We know from elementary quantum mechanics that the wave vector, $\vec{k}$ and the corresponding energies are quantized for a particle in a rigid box (Schiff 1968). However, the allowed $\vec{k}$ values are different for different shapes because of the boundary conditions. Also, the magnitude of $\vec{k}$ depends on the size of the box. We have calculated the allowed $\vec{k}$ values by imposing proper boundary conditions, depending on the shape and size of the dots. They are given as follows

$\vec{k}= \begin{cases}\frac{\pi}{2 R}\left(n_{x}, n_{y}, n_{z}\right)=\frac{\pi}{2 R} \vec{n} & \text { for cubic quantum dot, } \\ \frac{\pi}{\sqrt{3 R}}\left(n_{x}, n_{y}, n_{z}\right)=\frac{\pi}{\sqrt{3 R}} \vec{n} & \text { for spherical quantum dots. }\end{cases}$

Here $R$ is the radius of a spherical quantum dot and $L=2 R$ the length of the side of a cubical quantum dot. The discrete energy levels are then calculated for these $\vec{k}$ values and the smallest $\vec{n}$ is given by $(1,1,1)$ unlike $(0,0,0)$ in bulk. This is because $(0,0,0)$ will represent physically uninteresting situation in which $\psi_{\vec{k}}^{n}(\vec{r})=0$ everywhere in the box. Bandgap, $E_{\mathrm{g}}$, for cluster is the HOMO-LUMO gap at $\vec{n}=(1,1,1)$ denoted as $\Gamma$ point. The Hamiltonian matrix is diagonalized using typically $283 \mathrm{Gs}$ for each cluster. To assure the accuracy of the pseudo-potentials, we have calculated the complete band structure of the bulk semiconductors using the form factors given by Cohen and Bergstresser (1966). Here, the bulk bandgap is underestimated by $0.03 \mathrm{eV}$ for $\mathrm{ZnSe}$ and overestimated by $0 \cdot 19$ for $\mathrm{CdSe}$, respectively. We rigidly add these corresponding differences to the HOMO-LUMO gap of each cluster. Calculations for clusters are carried out till the bulk bandgap is achieved for larger clusters.

Effective mass approximation uses the particle-in-abox like approach with finite or infinite potential at the box boundaries. Efros and Efros (1982) applied EMA in limiting cases and have assumed the exciton to be confined in a spherically symmetric well bounded by infinitely high walls. Nosaka (1991) further modified this approach wherein a potential well of finite-depth, calculated from the electron affinity and the dielectric constant of the material and the surrounding medium, is used as suggested by Brus $(1983,1984)$. He has taken into account the Coulomb interaction between electron and hole generated due to the absorption of an incident photon, which was neglected in previous attempts. The Coulomb energy is estimated as the average value of Coulomb repulsion existing between an electron and a hole, assuming that both are in their $1 s$ state in the finite well. It becomes appreciable and comparable to the confinement energies for larger dots.

It was also pointed out that, though the electron-hole pair remains confined within the crystallite, some of their electric field penetrates into the matrix in which the crystallite is enclosed. This gives rise to small polarization term. Brus analytically obtained, $E_{x}(R)$, the excitation energy of lowest $1 s$ state for a spherical quantum dot of radius, $R$, as

$$
\begin{aligned}
E_{x}(R)= & E_{g}+\frac{\hbar^{2} \pi^{2}}{2 R^{2}}\left(\frac{1}{m_{\mathrm{e}}^{*}}+\frac{1}{m_{\mathrm{h}}^{*}}\right) \\
& -1.786 \frac{e^{2}}{\varepsilon_{1} R}-0.248 \frac{\mu e^{4}}{2 \varepsilon_{1}^{2} \hbar^{2}} .
\end{aligned}
$$

The second term in (15) is the kinetic energy, and the third term is Coulomb interaction between hole and electron. The fourth size-independent term is polarization energy in Ry (Symbols used have usual meaning).

To get higher level transition energies, the $l$-dependent radial Schrödinger equation can be solved assuming that the electron and hole are placed in various levels such as $\left(1 s_{\mathrm{e}}, 1 p_{\mathrm{h}}\right),\left(1 s_{\mathrm{e}}, 2 s_{\mathrm{h}}\right)$ etc. The eigenvalues and the corresponding eigen-functions of the radial Schrödinger equation thus calculated are used to evaluate the Coulomb energy, $E_{\mathrm{c}}$, by averaging the potential energy operator between the electron and the hole states. Thus

$$
\begin{gathered}
E_{\mathrm{c}}=-2\left(e^{2} / \varepsilon_{1}\right) \int_{0}^{\infty} r_{\mathrm{h}}^{2}\left[\int_{0}^{r_{\mathrm{h}}}\left\{\psi_{\mathrm{e}}^{2}\left(r_{\mathrm{e}}\right) \psi_{\mathrm{h}}^{2}\left(r_{\mathrm{h}}\right) / r_{\mathrm{h}}\right\} r_{\mathrm{e}}^{2} \mathrm{~d} r_{\mathrm{e}}+\right. \\
\left.\int_{r_{\mathrm{h}}}^{\infty} \psi_{\mathrm{e}}^{2}\left(r_{\mathrm{e}}\right) \psi_{\mathrm{h}}^{2}\left(r_{\mathrm{h}}\right) r_{\mathrm{e}} \mathrm{d} r_{\mathrm{e}}\right] d r_{\mathrm{h}},
\end{gathered}
$$

where $e$ is the electronic charge, $\varepsilon_{1}$ the dielectric constant of the material and $\psi_{\mathrm{e}}\left(r_{\mathrm{e}}\right), \psi_{\mathrm{h}}\left(r_{\mathrm{h}}\right)$ are the electron and hole radial wave-functions, respectively. The kinetic energy of conduction band electron, $T_{\mathrm{e}}$ and valence band hole, $T_{\mathrm{h}}$, can be written as $T_{i}=V_{0}-\left|E_{\mathrm{i}}\right|$, where $i=e$ or $h$ and $V_{0}$ the depth of the potential well and $E_{i}$ the respective eigen energy. HOMO-LUMO gap of the quantum dot, $E_{x}(R)$, can be written in terms of the bulk bandgap, $E_{\mathrm{g}}$, kinetic energies of the electron and hole, $T_{\mathrm{e}}$ and $T_{\mathrm{h}}$, respectively and the Coulomb energy, $E_{\mathrm{c}}$ 
Table 2. Input parameters used for EMA, finite well EMA and EPM calculations in the present study. $m_{\mathrm{e}}^{*}$ and $m_{\mathrm{h}}^{*}$ are effective masses of electron and hole, respectively. $\varepsilon_{1}$ and $\varepsilon_{2}$ are, respectively the dielectric constants of the material of the quantum dot, and of the liquid in which quantum dots are dispersed (water).

\begin{tabular}{|c|c|c|c|c|c|c|c|c|c|}
\hline \multirow[b]{2}{*}{ Material } & \multirow[b]{2}{*}{ Lattice constant } & \multirow[b]{2}{*}{$m_{\mathrm{e}}^{*}$} & \multirow[b]{2}{*}{$m_{\mathrm{h}}^{*}$} & \multirow[b]{2}{*}{ Electron affinity } & \multirow[b]{2}{*}{$\varepsilon_{1}$} & \multirow[b]{2}{*}{$\varepsilon_{2}$} & \multicolumn{3}{|c|}{ Bandgap } \\
\hline & & & & & & & Bulk* & EPM & Difference \\
\hline $\mathrm{CdSe}$ & $11 \cdot 43$ & $0 \cdot 13$ & 0.45 & $4 \cdot 58$ & $6 \cdot 20$ & 81 & $1 \cdot 73$ & $1 \cdot 92$ & $-0 \cdot 19$ \\
\hline GaAs & $10 \cdot 67$ & $0 \cdot 067$ & 0.62 & $4 \cdot 07$ & $13 \cdot 13$ & 81 & 1.43 & $1 \cdot 43$ & $0 \cdot 00$ \\
\hline
\end{tabular}

*Hartmann et al (1982)

$$
E_{x}(R)=E_{\mathrm{g}}+T_{\mathrm{e}}+T_{\mathrm{h}}+E_{\mathrm{c}} .
$$

We have also calculated the HOMO-LUMO gap, $E_{x}(R)$, for a quantum dot of radius, $R$, using (15), (16) and (17), to compare our EPM results with these simple approaches.

EMA works well only near band extrema and for an external potential which is slowly varying on the scale of the lattice constant. Further, in case of semiconductors, electrons and holes belong either to degenerate bands or to the bands which are close to each other on the energy scale. In such cases band mixing becomes important which single band EMA cannot account for. Multi-band EMA takes into account band mixing but not the nonparabolicity of bands. This is important in case of very small clusters where the effective masses may not be isotropic and may also differ significantly from the bulk values. In case of quantum dots, due to the finite size, the potential is not slowly varying over a distance of the order of electron wavelength. Thus, only an approximate estimate of the excitation energy can be made and the trend of its variation with size can be understood. One either needs to incorporate effects such as non-parabolicity of bands or resort to the empirical pseudo-potential or tight binding methods, which are applicable to both bulk and quantum dots.

To account for the excitonic binding energy, Coulombic attraction energy, $E_{\mathrm{c}}$, is numerically calculated with the electron and hole wave-functions calculated within the EPM. Thus the HOMO-LUMO gap of the quantum dot, $E_{x}(R)$, is written in terms of the bandgap, $E_{\mathrm{g}}$, obtained for the corresponding cluster from the matrix diagonalization at the $\Gamma$ point and the Coulomb energy, $E_{c}$, within the EPM, as given by (18)

$$
E_{x}(R)=E_{\mathrm{g}}(\text { of cluster })+E_{\mathrm{c}} .
$$

Table 2 shows various input parameters that are used in the present calculations. $\varepsilon_{1}$ and $\varepsilon_{2}$ are, respectively the dielectric constants of the material and of the medium (water) in which the dots are assumed to be dispersed. Columns 8 and 9 display, respectively the reported bulk bandgaps and those calculated by our EPM code and the last column shows the difference of the two.

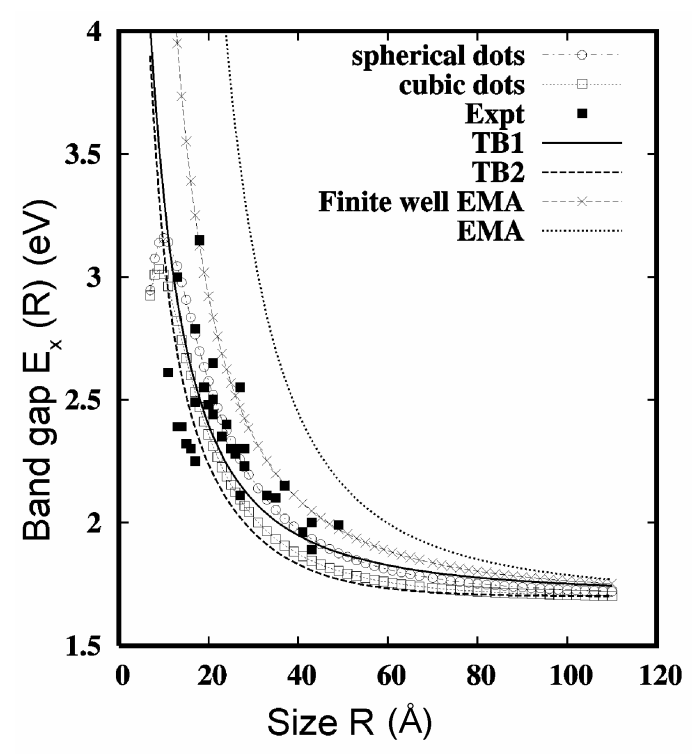

Figure 2. Variation of HOMO-LUMO gap with size for CdSe quantum dots. Spherical and cubic dots are the present EPM results for dots of corresponding shapes. TB1 and TB2 refer to the empirical fits to the tight binding results suggested by Sapra and Sarma (2004) and Viswanatha et al (2005), respectively. Experimental results are from Murray et al (1993), Kuno et al (1997), Bowen Katari et al (1994), Rogach et al (1999), Murray et al (1993), Colvin et al (1994) and Shim and Phillippe (1999).

\section{Results and discussion}

\subsection{HOMO-LUMO gap variation with size}

Figures $2-4$ show the excitonic energies calculated by us within effective mass approximation with infinite potential (EMA) using (15), EMA with finite confining potential (Finite well EMA) using (17) and EPM results for cubic (cubic dots) and spherical (spherical dots) shapes using (18) compared with the experimentally observed values and calculated by the tight binding method by Sapra and Sarma (2004) (TB1) and Viswanatha et al (2005) (TB2) for CdSe, $\mathrm{ZnSe}$ and GaAs quantum dots, respectively. All the curves qualitatively show the correct trend viz. an increase in the HOMO-LUMO gap with de- 
creasing dot size. However, as expected EMA highly overestimates excitonic energies in the region of interest. Inclusion of Coulomb energy and finite depth potential considerably lowers the excitonic energy and the results are found to be in good agreement with experiments for medium sized clusters, as opposed to EMA. In small crystallites the lowest energy eigenvalues lie in the region

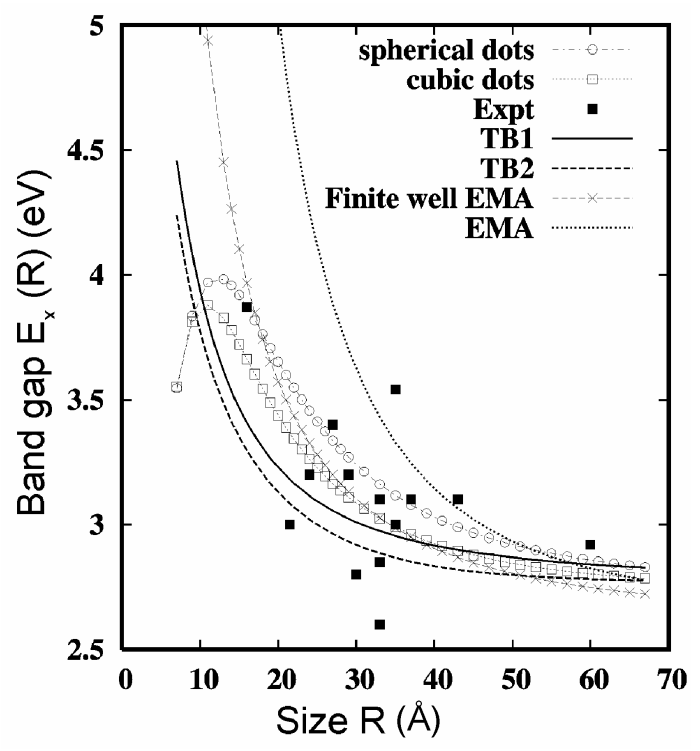

Figure 3. Variation of HOMO-LUMO gap with size for $\mathrm{ZnSe}$ quantum dots. Symbols used are same as in figure 2. Experimental results are from Chestnoy et al (1987), Kumbhojkar et al (1997), Leppert et al (1997a, b) and Hines and Guyot-Sionnest (1998).

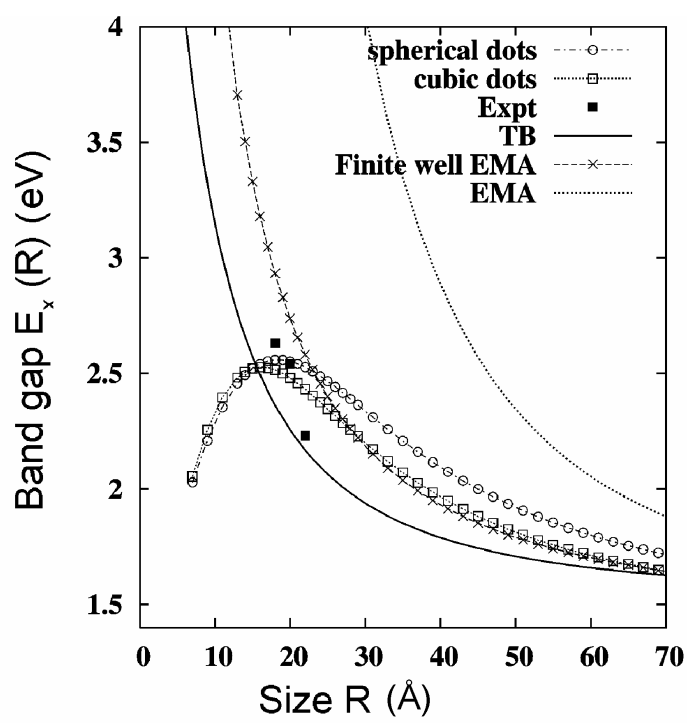

Figure 4. Variation of HOMO-LUMO gap with size for GaAs quantum dots. TB refers to the empirical fit to the tight binding results suggested by Viswanatha et al (2005). Other symbols used are same as in figure 2. Experimental results are from Olshavsky et al (1990) and Malik et al (2003). of energy bands where they are no longer parabolic. Thus the assumption of the bands being parabolic in case of both the finite and infinite well EMA breaks down and introduces significant error. EMA fails when kinetic energy of the lighter particle becomes substantial, and it overestimates the kinetic energy in small bandgap materials when surface charge density is important.

One of the additional aspects of quantum confinement effect is the bandgap dependence on the physical shape of the quantum dots. The exciton energies for spherical quantum dots are found to be in good agreement with the observed values over a broad size range. Thus our theoretical study shows that the crystallites prefer to be spherical in shape. This is consistent with the experimental observations. The crystallites having sizes $>10 \AA$ are known to exist in spherical shape. The kinetic energy is underestimated in cubic shape by virtue of the fact that the kinetic energy of a particle confined in a sphere of radius, $R$, is greater than that of a particle confined in a cubic box of length, $L=2 R$. The empirical pseudo-potential values for spherical quantum dots show an excellent agreement with experiments in case of CdSe. The EMA and finite well EMA curves are also shown for comparison. A comparison with the TB results of Sapra and Sarma (2004) and Viswanatha et al (2005) shows that their curves show the trends like our results for cubic dots and spherical dots, respectively for $\mathrm{CdSe}$. However, a close look reveals that TB results underestimate the values slightly.

The experimental data for ZnSe show a large scatter and therefore, a comparison with the model also has less predictive power. For $\mathrm{ZnSe}$, the TB results underestimate the gap in comparison to EPM results and are in disagreement with experiments. We discuss the possible reason for this disagreement after discussing all the results.

Lattice generally contracts by a small amount in quantum dots. We have incorporated such observed lattice contraction in case of $\mathrm{ZnSe}$. Table 3 shows that excitation energies calculated using lattice contraction (LC) of $2.5 \%$ for ZnSe quantum dots, of typical observed sizes, tend toward the experimental values. The inherent experimental polydispersity of the samples is an important factor responsible for the observed discrepancy. EMA and finite well EMA, being too simple, fail to include the effects of lattice contraction.

It is noteworthy that EPM calculation shows a 'redshift' for very small clusters instead of a monotonic blueshift. Similar observations are reported for GaAs (Rama Krishna and Friesner 1991a, b) and silicon (Rama Krishna and Friesner 1992). Such a red-shift was also predicted by the PM3 semi-empirical calculation carried out on DMSO capped CdS clusters of $\mathrm{Cd}_{6} \mathrm{~S}_{6}(3.53 \mathrm{eV})$ and $\mathrm{Cd}_{3} \mathrm{~S}_{3}$ (3.35 eV) (Robbles 1999). This may be an artifact of the empirical potentials used in the calculations. More experimental data is needed in very small size regime where theoretically a red-shift has been predicted in the absorption. It should be mentioned that the HOMO-LUMO gap 
Table 3. HOMO-LUMO gap calculated with experimental lattice contraction for ZnSe quantum dots (Kumbhojkar et al 1997).

\begin{tabular}{lcccccc}
\hline & \multicolumn{4}{c}{ EPM } & \\
\cline { 2 - 3 } & \multicolumn{2}{c}{ Without lattice contraction } & & \multicolumn{2}{l}{ With lattice contraction } & \\
\cline { 2 - 3 } Dia. $(\AA)$ & Cubic & Spherical & & Cubic & Spherical & Exptl. \\
\hline 24 & 3.26 & 3.45 & & 3.04 & 3.24 & 3.20 \\
32 & 3.04 & 3.19 & & 2.79 & 2.95 & 2.60 \\
\hline
\end{tabular}
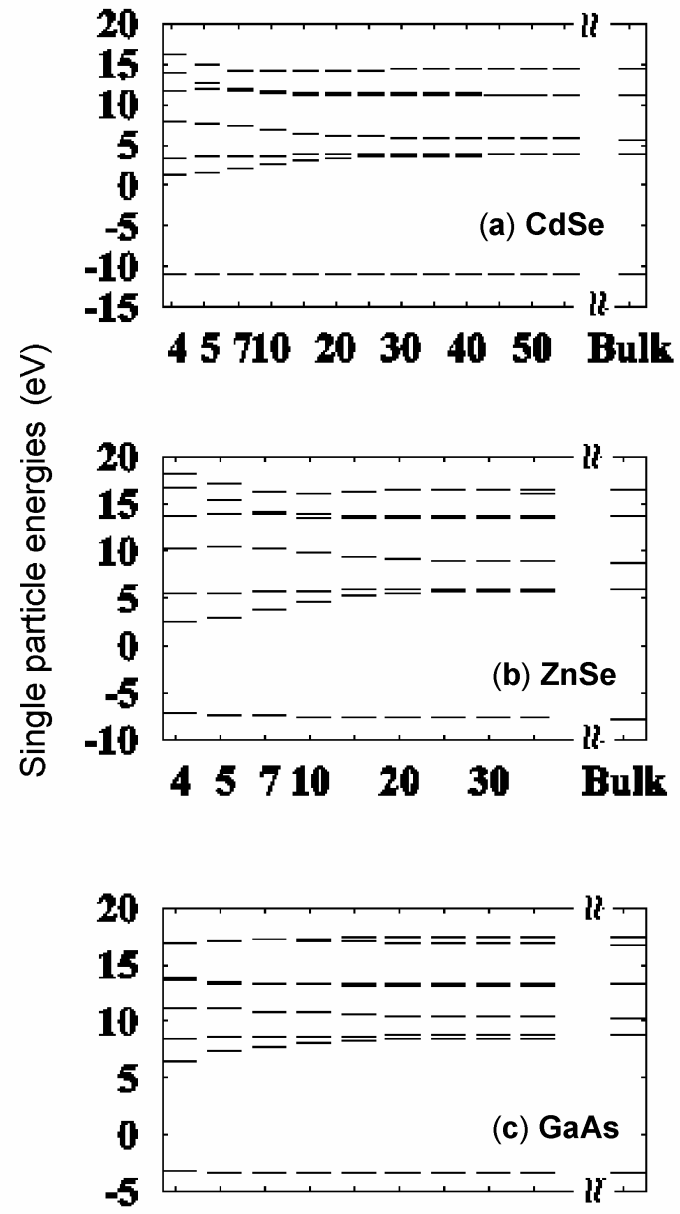

$\begin{array}{llllll}10 & 20 & 30 & 40 & 50 & \text { Bulk }\end{array}$

Size of dots $(\AA)$

Figure 5. Single particle energy levels at $\Gamma$ point for various sizes of quantum dots of (a) CdSe, (b) ZnSe and (c) GaAs, respectively.

for smaller sizes is of course larger than the bulk bandgap; i.e. it is blue-shifted with respect to the bulk, as expected. In this size regime, the lattice is distorted considerably and these distortion effects, which may be neglected otherwise, are prominent. If we assume the same geometry as that of the bulk for dots of sizes $\sim 5 \AA$, even one unit cell is not accommodated and the assumption of periodicity breaks down. Such dots are known to be pyramidal (Dance et al 1984) or elliptic in shape (Vossmeyer et al 1994).

\subsection{Electronic structure of quantum dots}

Empirical pseudo-potential method is used to calculate the electron energies at various allowed high symmetry points in the first Brillouin zone of the reciprocal lattice. Figures 5(a)-(c) show the electronic levels at $\Gamma$ points of clusters having different radii. Bulk energies at $\Gamma$ are also shown in each of these figures for comparison.

The HOMO-LUMO gap clearly has a tendency to decrease with increasing size and it finally converges to bulk bandgap. A gradual upward shift in HOMO and downward shift in LUMO energies can be clearly seen with increase in size for $\mathrm{CdSe}$ and $\mathrm{ZnSe}$. These energy diagrams depict the difference in the nature of energy spectrum for small and large clusters. For small clusters the energies are more molecular-like whereas as we move, with increasing size a band forming tendency is seen. As we approach the bulk limit, the structure and coordination number change which in turn affect the symmetry (degeneracy) of the bands. The nature of single-particle states is thus different. Our study predicts that the deep lying $d$-states are not affected by size quantization in II-VI semiconductors. These states are widely separated from the highest valence band originating from $s$ and $p$ states of the constituent atoms.

We have extended our studies to III-V semiconductors viz. GaAs, which is of technological importance. We present the results of variation in HOMO-LUMO gap with size for GaAs in figure 4. It is found that the results of EPM for cubic dots match with finite well EMA results for sizes greater than $25 \AA$. Also, a comparatively larger 'red-shift' is found for GaAs which can be understood if we look at the single particle energy levels at the $\Gamma$ point, shown in figure 5(c). For GaAs, only few experimental results are available and they do not agree with each other. For III-V semiconductors also TB underestimates the gap in comparison to EPM results. In GaAs, the highest valence band is not so flat as in II-VI semiconductors. Secondly, the lowest conduction band energies decrease as one moves from $\Gamma$ to $L$ point along the $\Lambda$ direction. 
This results into an increase in HOMO and a decrease in LUMO energies with size for cluster radius up to $\approx 10 \AA$. Also the separation between the higher valence molecular orbitals consisting of $s$ - and $p$-like states is as much as between the lower $d$-like states. Hence, the $d$-states have to be included in the calculation for very small sizes.
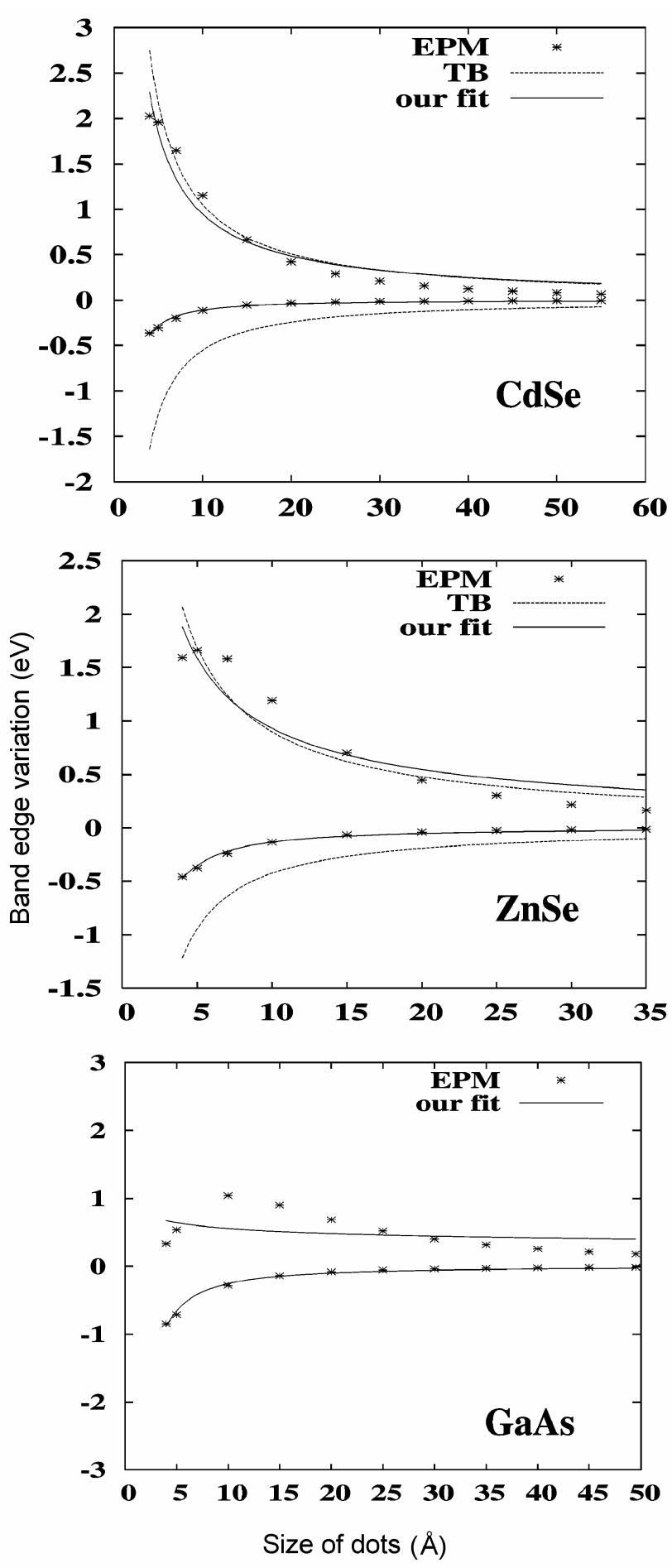

Figure 6. Band edge variation with size of dot.
As mentioned by Sapra and Sarma (2004), the bandgap variation is determined by shifts in the top of the valence band (TVB) and bottom of the conduction band (BCB) as a function of dot size. Such shifts of the band edges go to zero for large sized dots. The shifts for BCB are in general larger than the TVB for any particular sized dot. Hence, the bandgap is dominantly determined by the BCB or the LUMO. This larger shift is attributed to the smaller effective mass of the electron in comparison to the effective hole mass. Sapra and Sarma (2004) have fitted the calculated band edge shifts, $\Delta E_{i}^{\text {edge }}$, to vary inversely with some power of $d$, the diameter of the quantum dot. They have seen that this variation is slower than the $d^{-2}$ behaviour predicted by EMA.

Following these workers, we have also carried out a similar fit for dots of both the spherical and cubic shapes. The shift in band edge has been fitted with (19) as given below

$$
\Delta_{i}^{\text {edge }}=\frac{a_{i}}{d^{b_{i}}}
$$

where $d$ is the diameter of the dot and $i=h$ for TVB and $i=e$ for $\mathrm{BCB}$. The variation has been displayed in figure 6 and our fitted parameters are listed in table 4 . Our values for the parameters, $a_{\mathrm{i}}$ and $b_{\mathrm{e}}$, are numerically smaller than TB results and for $b_{\mathrm{h}}$ are numerically larger for $\mathrm{CdSe}$ and $\mathrm{ZnSe}$ quantum dots. The fit for band edge variation for the conduction band is similar to the TB result and does not match with the data points. This indicates that the electron effective mass may be different from the bulk value since it changes a lot with size of the dot. The variation in TVB is slower than the TB results.

TB method is supposed to be computationally less demanding and provides a description in terms of atomic orbitals and hopping interactions. Thus intuitive understanding of the system is available. Sapra and Sarma (2004) have used a realistic $s p^{3} d^{5}$ basis for anions and cations and $s p^{3}$ basis for the cations, with inclusion of next nearest neighbour interaction. They vary the hopping integrals and on-site energies to match the TB results with first principles results for band structure of corresponding bulk material. It is, however, not sufficient to get the energies at various symmetry points accurately described by the TB method; the curvature of the bands is also important, in particular, the curvature of highest valence band and lowest conduction band need to be determined accurately as they decide the effective masses of hole and electron, respectively. Secondly the quantum dots may

Table 4. Values of parameters $a_{i}$ and $b_{i}$ used in (19).

\begin{tabular}{lrcrc}
\hline Material & $a_{\mathrm{e}}$ & $b_{\mathrm{e}}$ & $a_{\mathrm{h}}$ & $b_{\mathrm{h}}$ \\
\hline CdSe & 16.97 & 0.96 & -6.60 & 1.37 \\
ZnSe & 9.31 & 0.77 & -8.73 & 1.40 \\
GaAs & 1.04 & 0.21 & -15.27 & 1.37 \\
\hline
\end{tabular}


not retain the same geometrical structure as that of bulk. Hence, the TB parameters like hopping integrals and onsite energies are not transferable. Transferability requires a more elaborate fitting for various hypothetical structures and many possible near neighbour distances. Thus although in the work of Sapra and Sarma (2004), the agreement with experiments is reasonable with inclusion of $d^{5}$ orbitals in the TB basis and inclusion of next nearest neighbour interaction in the TB Hamiltonian, better description is possible within TB formalism with elaborate fitting. Thirdly, the dependence of the Coulomb interaction term, $E_{c}$, is taken to be $1 / R$ type, as described within EMA. We have found out that decrease in $E_{\mathrm{c}}$ is slower than $1 / R$ when the actual electron and hole wave-functions described within the independent particle approximation are used to evaluate the average Coulomb energy. This, of course, cannot take into account the many body effects to match with the excitonic energies but it does indicate why the TB results provide an underestimation in comparison to EPM. An in-depth comparison of excitation energies calculated by these techniques and experiments for all the II-VI semiconductor quantum dots and some III-V semiconductor quantum dots has been reported elsewhere (Kumbhojkar et al 2000, 2001, 2002).

\section{Conclusions}

We have studied quantum confinement effects, observed in II-VI and proto-type III-V semiconducting quantum dots, by applying the empirical theories. The excitation energies were found to be size and shape dependent. Our EPM results show a better agreement with experimentally observed HOMO-LUMO gaps than infinite and finite well potential EMA calculations, over the entire size range studied. Structural changes such as lattice contraction, play a crucial role in such studies and EPM is shown to be capable of incorporating these. Average Coulomb energy is also calculated fairly well within EPM.

Excitonic energies calculated by these empirical methods deviate appreciably for very small clusters possibly due to non-parabolicity of bands and a difference in potentials due to structural changes in this size regime. Also the bulk parameters such as effective masses of electron and hole may be different than in bulk. In case of very small clusters a red-shift is predicted by EPM, which lacks experimental data for confirmation. Use of $a b$ initio potentials may help to resolve this problem. Our results have been compared with the other theoretical results available in the literature like those using TB methods and the possible reasons for disagreement wherever existed have been discussed.

We have successfully shown that empirical pseudopotential method can predict the trends in electronic structure evolution from small clusters to bulk. Small clusters have molecular-like energy levels and with sub- stantial increase in size, the band forming tendency is clearly seen. LUMO energy decreases whereas HOMO energy increases, comparatively slowly, as size increases, for II-VI semiconductor dots; finally the difference between them converging to bulk bandgap. In III-V semiconductor dots, for very small sizes, there is a substantial deviation from such trend. Empirical pseudo-potential is applied to calculate the electronic structure at high symmetry points. A band forming tendency is also seen at energy levels away from LUMO.

\section{Acknowledgement}

The authors acknowledge the financial support from the Department of Science and Technology, Government of India.

\section{References}

Bowen Katari J E, Colvin V L and Alivisatos A P 1994 J. Phys. Chem. 984109

Brus L E 1983 J. Chem. Phys. 795566

Brus L E 1984 J. Chem. Phys. 804403

Chestnoy N, Hull R and Brus L E 1987 J. Chem. Phys. 85 2237

Cohen M L and Heine V 1970 Solid state physics (eds) H Eherenreich et al (New York: Academic Press) Vol. 24, p. 37

Cohen M L and Chelikowsky J R 1989 Electronic structure and optical properties of semiconductors (Berlin: SpringerVerlag)

Cohen M L and Bergstresser T K 1966 Phys. Rev. 141789

Colvin V L, Schlamp M C and Alivisatos A P 1994 Nature 370 354

Dance I G, Choy A and Shudder M L 1984 J. Am. Chem. Soc. 1066285

Éfros Al L and Éfros A L 1982 Sov. Phys. Semicond. 16772

Eichkorn K and Ahlrichs R 1998 Chem. Phys. Lett. 288235

Harrison W A 1966 Pseudopotentials in the theory of metals (New York: Benjamin)

Hartmann H, Mach R and Selle B 1982 Current topics in materials science (ed.) E Kaldis (Amsterdam: North-Holland Pub. Co.) 9

Hines H and Guyot-Sionnest P 1998 J. Phys. Chem. B102 3655

Joswig Jan-Ole, Springborg M and Seifert G 2000 J. Phys. Chem. B104 2617

Kayanuma Y 1988 Phys. Rev. B38 9797

Kayanuma Y and Momiji H 1990 Phys. Rev. B41 10261

Kumbhojkar N 2002 Studies of size dependence of electronic structure of semiconducting quantum dots, Ph.D. Thesis, University of Pune, Pune

Kumbhojkar N and Kshirsagar A 2001a Proceedings of national conference on 'science and technology of nanomaterials and clusters' (New Delhi: Allied Publishers Limited)

Kumbhojkar N and Kshirsagar A 2001b in Science and technology of nano-structured materials (eds) B K Rao et al (NY: Nova Publishers)

Kumbhojkar N, Mahamuni S, Leppert V and Risbud S H 1997 Nanostruct. Mater. 10117 
Kumbhojkar N, Nikesh V V, Kshirsagar A and Mahamuni S 2000 J. Appl. Phys. 886260

Kuno M, Lee J K, Dabbousi B O, Mikulec F V and Bawendi M G 1997 J. Chem. Phys. 1069869

Lee In-Ho, Kim Yong-Hoon and Ann Kang-Hun 2001 J. Phys.: Condens. Matter 131987

Leppert V, Mahamuni S, Kumbhojkar N and Risbud S H 1997a Mater. Sci. Eng. B52 89

Leppert V, Risbud S H and Fendrof M 1997b Philos. Mag. Lett. 7529

Leung K, Pokrant S and Whaley K B 1998 Phys. Rev. B57 12291

Lippens P E and Lannoo M 1989 Phys. Rev. B39 10935

Malik M A, O'Brien P, Noragev S and Smith J 2003 J. Mater. Chem. 132591

Matxain J M, Irigoras A, Fowler J E and Ugalde J M 2001 Phys. Rev. A63 13202

Matxain J M, Irigoras A, Fowler J E and Ugalde J M 200164 13201

Murray C B, Nirmal N, Norris D J, Bawendi M G and Shank C V 1993 Z. Phys. D26 231

Murray C B, Norris D J and Bawendi M G 1993 J. Am. Chem. Soc. 1158706

Nair S V, Sinha S and Rustagi K C 1987 Phys. Rev. B35 4098

Nair S V, Ramaniah L M and Rustagi K C 1992 Phys. Rev. B45 5969

Nosaka Y 1991 J. Phys. Chem. 955054

Olshavsky M A, Goldstein A N and Alivisatos A P $1990 \mathrm{~J} . \mathrm{Am}$. Chem. Soc. 1129438

Pickett W E 1989 Comput. Phys. Rep. 9115 and references therein

Pokrant S and Whaley K B 1999 Eur. Phys. J. D6 255

Ramaniah L M and Nair S V 1993 Phys. Rev. B47 7132
Ramaniah L M and Nair S V 1995 Physica B212 245

Rama Krishna M V and Friesner R A 1991a J. Chem. Phys. 95 8309

Rama Krishna M V and Friesner R A 1991b Phys. Rev. Lett. 67 629

Rama Krishna M V and Friesner R A 1992 J. Chem Phys. 96 873

Robbles J, Mayorga O, Lee T and Diaz D 1999 Nanostruct. Mater. 11283

Rogach A L, Kornowski A, Gao M, Echyüller A E and Weller H 1999 J. Phys. Chem. B103 3065

Ruppin R 1989 J. Phys. Chem. Solids 50877

Sapra S and Sarma D D 2004 Phys. Rev. B69 125304

Schiff L I 1968 Quantum mechanics (Tokyo: McGraw-Hill Ltd.) p. 39

Sercel C and Vahala K J 1990 Phys. Rev. B42 3690

Shim M and Phillippe Guyot-Sionnest 1999 J. Chem. Phys. 11 6955

Slater J C and Koster G F 1954 Phys. Rev. 941498

Tran Thoi D B, Hu Y Z and Koch S W 1990 Phys. Rev. B42 11261

Troparevsky M Claudia and Chelikowsky J R 2001 J. Chem. Phys. 114943

Vasiliev I, Ögüt S and Chelikowsky J R 1999 Phys. Rev. B60 R8477

Viswanatha R, Sapra S, Saha-Dasgupta T and Sarma D D 2005 Phys. Rev. B72 045333

Vossmeyer T, Katsikas L, Giersig M, Popovic I G, Diesner K, Chemseddine A, Eychmuller A and Weller H 1994 J. Phys. Chem. 987665

Wang Y and Herron N 1990a J. Phys. Chem. 924988

Wang Y and Herron N 1990b Phys. Rev. B42 7253

Yoffe A D 1993 Adv. Phys. 42173 and references therein 